\title{
Total Unidominating Functions of a Path
}

\author{
V. Anantha Lakshmi \\ Department of Mathematics \\ P.R.Govt. College (A), Kakinada-533001, \\ Andhra Pradesh, India
}

\author{
B. Maheswari \\ Department of Applied Mathematics \\ S.P.Women's University, Tirupati-517502, \\ Andhra Pradesh, India
}

\begin{abstract}
Domination in graphs is an emerging area of research at present and it has applications to several fields such as School bus routing, Computer communication networks, Facility location problems, Locating radar stations problem etc. Recently dominating functions in domination theory have received much attention. The new concepts viz., unidominating functions and total unidominating functions have been introduced. In this paper some total unidominating functions of a path are presented and determined its total unidomination number. Also the number of total unidominating functions with minimum weight is investigated.
\end{abstract}

\section{Keywords}

Path, Total unidominating function, Total unidomination number.

\section{INTRODUCTION}

Graph Theory has a wide range of applications in engineering, physical, biological and social sciences and other branches of Mathematics. The domination in graphs is one of the concepts in graph theory which has attracted many researchers to work on it. Allen R.B. and Laskar.R. [1], Cockayne E.J. and Hedetniemi S.T.[3] have contributed significantly to the theory of domination. Domination and its properties have been extensively studied by T.W.Haynes and others in [6], [7]. Total dominating sets are introduced by Cockaane et.al. [4]. The concept of total dominating functions, were introduced by Cockayne et.al. [5]. The authors have introduced the new concepts unidominating and total unidominating functions and studied unidominating function for paths [2]. In this paper the total unidominating functions of a path are studied and obtained total unidomination number and the number of total unidominating functions with minimum weight. Further the results obtained are illustrated.

\section{TOTAL UNIDOMINATING FUNCTIONS AND TOTAL UNIDOMINATION NUMBER}

In this section the concepts of total unidominating function and total unidomination number of a graph are introduced as follows.

Definition 2.1: Let $G(V, E)$ be a connected graph. A function $f: V \rightarrow\{0,1\}$ is said to be a total unidominating function if

$$
\begin{aligned}
& \sum_{u \in N(v)} f(u) \geq 1 \forall v \in V \text { and } f(v)=1, \\
& \sum_{u \in N(v)} f(u)=1 \forall v \in V \text { and } f(v)=0 .
\end{aligned}
$$

Definition 2.2: The total unidomination number of a connected graph $G(V, E)$ is defined as

$\min \{f(V) / f$ is a total unidominating function $\}$.

where $f(V)=\sum_{u \in V} f(u)$ is called as the weight of the total unidominating function $f$.

It is denoted by $\gamma_{t u}(G)$.

\section{TOTAL UNIDOMINATION NUMBER OF A PATH}

In this section the results on total unidomination number of a path are proved and the number of total unidominating functions withminimum weight are found. Further the results obtained are illustrated.

Theorem 3.1: The total unidomination number of a path $P_{n}$ is

$\gamma_{t u}\left(P_{n}\right)=\left\{\begin{array}{l}\left\lceil\frac{n}{2}\right\rceil \quad \text { if } n \equiv 0,1,3(\bmod 4) \\ \left\lceil\frac{n}{2}\right\rceil+1 \quad \text { if } n \equiv 2(\bmod 4)\end{array}\right.$

Proof: Let $P_{n}$ be a path with vertex set $V=\left\{v_{1}, v_{2}, \ldots, v_{n}\right\}$, $n \geq 2$.

To find the total unidomination number of $P_{n}$, the following four cases arise.

Case 1: Let $n \equiv 0(\bmod 4)$.

Define a function $f: V \rightarrow\{0,1\}$ by

$f\left(v_{i}\right)= \begin{cases}1 & \text { for } i \equiv 2,3(\bmod 4), \\ 0 & \text { for } i \equiv 0,1(\bmod 4) .\end{cases}$

Now the condition for total unidominating function at every vertex is checked in various subcases as follows.

Sub case 1: Let $i \equiv 0(\bmod 4)$ and $i \neq n$. Then $f\left(v_{i}\right)=0$. Now $\sum_{u \in N\left(v_{i}\right)} f(u)=f\left(v_{i-1}\right)+f\left(v_{i+1}\right)=1+0=1$.

If $i=n$ then $\sum_{u \in N\left(v_{n}\right)} f(u)=f\left(v_{n-1}\right)=1$.

Sub case 2 : Let $i \equiv 1(\bmod 4)$ and $i \neq 1$. Then $f\left(v_{i}\right)=0$.

Now $\sum_{u \in N\left(v_{i}\right)} f(u)=f\left(v_{i-1}\right)+f\left(v_{i+1}\right)=0+1=1$. 
If $i=1$ then $\sum_{u \in N\left(v_{1}\right)} f(u)=f\left(v_{2}\right)=1$.

Sub case 3: let $i \equiv 2(\bmod 4)$. Then $f\left(v_{i}\right)=1$.

Now $\sum_{u \in N\left(v_{i}\right)} f(u)=f\left(v_{i-1}\right)+f\left(v_{i+1}\right)=0+1=1$.

Sub case 4: Let $i \equiv 3(\bmod 4)$. Then $f\left(v_{i}\right)=1$.

Now $\sum_{u \in N\left(v_{i}\right)} f(u)=f\left(v_{i-1}\right)+f\left(v_{i+1}\right)=1+0=1$.

Since $\sum_{u \in N\left(v_{i}\right)} f(u)=1$ forf $\left(v_{i}\right)=1$ and $\sum_{u \in N\left(v_{i}\right)} f(u)$

$$
=1 \operatorname{for} f\left(v_{i}\right)=0 \text {, }
$$

it follows that $f$ is a total unidominating function.

Now $\begin{aligned} \sum_{u \in V} f(u)=\underbrace{0+1+1+0}+\cdots+\underbrace{0+1+1+0} & =2 \cdot \frac{n}{4}\end{aligned}$

Hence by the definition of total unidomination number,

$\gamma_{t u}\left(P_{n}\right) \leq \frac{n}{2}---(1)$

If $f$ is a total unidominating function of $P_{n}$, then it can be seen that amongst four consecutive vertices at most two vertices can have functional value 0 and at least two vertices must have functional value 1 .

Therefore sum of the functional values of four consecutive vertices is greater than or equal to 2 .

That is $\sum_{i=1}^{4} f\left(v_{i}\right) \geq 2, \sum_{i=5}^{8} f\left(v_{i}\right) \geq 2, \ldots, \sum_{i=n-3}^{n} f\left(v_{i}\right) \geq 2$.

Therefore $\sum_{u \in V} f(u)=\sum_{i=1}^{4} f\left(v_{i}\right)+\sum_{i=5}^{8} f\left(v_{i}\right)+\cdots$

$$
+\sum_{i=n-3}^{n} f\left(v_{i}\right) \geq \underbrace{2+2+\cdots+2}_{\frac{n}{4} \text { times }} \geq \frac{n}{2} .
$$

This is true for any total unidominating function.

Therefore

$\min \{f(V) /$ fisatotalunidominatingfunction $\} \geq \frac{n}{2}$.

Thus $\gamma_{t u}\left(P_{n}\right) \geq \frac{n}{2}---(2)$

Therefore from the inequalities (1) and (2) $\gamma_{t u}\left(P_{n}\right)=\frac{n}{2}$.

Case 2: Let $n \equiv 1(\bmod 4)$.

Define a function $f: V \rightarrow\{0,1\}$ by

$$
f\left(v_{i}\right)=\left\{\begin{array}{lr}
1 & \text { for } i \equiv 2,3(\bmod 4), \\
0 & \text { for } i \equiv 0,1(\bmod 4), i \neq \mathrm{n}-1,
\end{array}\right.
$$

and $f\left(v_{n-1}\right)=1$.

As in similar lines of Case 1 the condition of total unidominating function at the vertices $v_{1}, v_{2}, \ldots \ldots, v_{n-3}$ can be verified. Now the condition of total unidominating function at the vertices $v_{n-2}, v_{n-1}$ and $v_{n}$ is checked as follows.

$$
\begin{aligned}
& \text { If } i=n-2 \text { then } \sum_{u \in N\left(v_{i}\right)} f(u)=f\left(v_{n-3}\right)+f\left(v_{n-1}\right) \\
& =1+1=2>1 .
\end{aligned}
$$

$$
\begin{aligned}
& \text { If } i=n-1 \text { then } \sum_{u \in N\left(v_{i}\right)} f(u)=f\left(v_{n-2}\right)+f\left(v_{n}\right) \\
& =1+0=1 . \\
& \text { If } i=n \text { then } \sum_{u \in N\left(v_{n}\right)} f(u)=f\left(v_{n-1}\right)=1 .
\end{aligned}
$$

It follows that $f$ is a total unidominating function.

Now $f(V)=\sum_{u \in V} f(u)$

$=\underbrace{0+1+1+0}+\cdots+\underbrace{0+1+1+0}+\underbrace{0+1+1+1+0}$

$=2\left(\frac{n-5}{4}\right)+3=\frac{n+1}{2}=\left\lceil\frac{n}{2}\right\rceil$.

Hence by the definition of total unidomination number,

$\gamma_{t u}\left(P_{n}\right) \leq\left\lceil\frac{n}{2}\right\rceil---(1)$

Now $n \equiv 1(\bmod 4) \Rightarrow n-5 \equiv 0(\bmod 4)$.

Then for any total unidominating function $f$,

as in Case 1, we have $\sum_{i=1}^{n-5} f\left(v_{i}\right) \geq \frac{n-5}{2}$.

To get the weight of $f$ as minimum weight, the functional values are assigned in such a way that

$\sum_{i=1}^{n-5} f\left(v_{i}\right)=\frac{n-5}{2}$.

The possibility for assigning functional values to the remaining five vertices say $v_{n-4}, v_{n-3}, v_{n-2}, v_{n-1}, v_{n}$ is $0,1,1,1,0$.

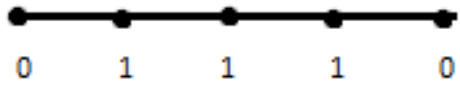

$$
\begin{aligned}
& \text { Now } \begin{aligned}
& \sum_{i=n-4}^{n} f\left(v_{i}\right)=3 \text {. } \\
& \text { So } \quad \sum_{u \in V} f(u)=\sum_{i=1}^{n-5} f\left(v_{i}\right)+\sum_{i=n-4}^{n} f\left(v_{i}\right)=\frac{n-5}{2}+3 \\
&=\frac{n+1}{2}=\left\lceil\frac{n}{2}\right\rceil .
\end{aligned}
\end{aligned}
$$

Therefore for any total unidominating function $f$, $\sum_{u \in V} f(u) \geq\left\lceil\frac{n}{2}\right\rceil$.

Thus $\min \{f(V) / f$ is a total unidominating function $\} \geq\left\lceil\frac{n}{2}\right\rceil$.

That is $\gamma_{t u}\left(P_{n}\right) \geq\left\lceil\frac{n}{2}\right\rceil---(2)$

Therefore from the inequalities (1) and (2) $\gamma_{t u}\left(P_{n}\right)=\left\lceil\frac{n}{2}\right\rceil$.

Case 3: Let $n \equiv 2(\bmod 4)$.

Sub case 1: Let $n=2$.

Then clearly a function defined by $f\left(v_{1}\right)=1, f\left(v_{2}\right)=1$ is the only total unidominating function and

$\gamma_{t u}\left(P_{2}\right)=\left\lceil\frac{2}{2}\right\rceil+1=2$.

Sub case 2: Let $n \geq 6$.

Define a function $f: V \rightarrow\{0,1\}$ by 
$f\left(v_{i}\right)=\left\{\begin{array}{lr}1 & \text { for } i \equiv 2,3(\bmod 4), \quad i \neq n, \\ 0 & \text { for } i \equiv 0,1(\bmod 4), i \neq \mathrm{n}-1, \mathrm{n}-2,\end{array}\right.$

and $f\left(v_{n-2}\right)=f\left(v_{n-1}\right)=1, f\left(v_{n}\right)=0$.

It can be verified in similar lines as in Case 1 that $f$ is a total unidominating function.

$$
\text { Now } \begin{aligned}
\sum_{u \in V} f(u)= & \underbrace{0+1+1+0}+\cdots+\underbrace{0+1+1+0} \\
& +\underbrace{0+1+1+1+1+0} \\
& =2\left(\frac{n-6}{4}\right)+4=\frac{n}{2}+1 .
\end{aligned}
$$

Therefore $\gamma_{t u}\left(P_{n}\right) \leq \frac{n}{2}+1---(1)$

If $f$ is any total unidominating function with minimum weight, then the functional values of first three vertices and the last three vertices must satisfy the following condition.

$$
\sum_{i=1}^{3} f\left(v_{i}\right)=2 \text { and } \sum_{i=n-2}^{n} f\left(v_{i}\right)=2
$$

Now $n \equiv 2(\bmod 4) \Rightarrow n-6 \equiv 0(\bmod 4)$. We have seen in Case 1 that for any totalunidominating function $f$,

$\sum_{u \in V} f(u) \geq \frac{n}{2}$.

Therefore for the $n-6$ vertices from $v_{4}$ to $v_{n-3}$, we have

$\sum_{i=4}^{n-3} f\left(v_{i}\right) \geq \frac{n-6}{2}$.

Therefore $\sum_{u \in V} f(u)=\sum_{i=1}^{3} f\left(v_{i}\right)+\sum_{i=4}^{n-3} f\left(v_{i}\right)+\sum_{i=n-2}^{n} f\left(v_{i}\right)$

$$
\geq 2+\frac{n-6}{2}+2 \geq \frac{n+2}{2} \geq \frac{n}{2}+1 \text {. }
$$

Thus $\gamma_{t u}\left(P_{n}\right) \geq \frac{n}{2}+1---(2)$

Therefore from the inequalities (1) and (2),

$\gamma_{t u}\left(P_{n}\right)=\frac{n}{2}+1=\left\lceil\frac{n}{2}\right\rceil+1$.

$\left(n \equiv 2(\bmod 4) \Rightarrow n\right.$ is even $\Rightarrow \frac{n}{2}=\left\lceil\frac{n}{2}\right\rceil$.)

Case 4: Let $n \equiv 3(\bmod 4)$.

Define a function $f: V \rightarrow\{0,1\}$ by

$f\left(v_{i}\right)= \begin{cases}1 & \text { for } i \equiv 2,3(\bmod 4), \\ 0 & \text { fori } \equiv 0,1(\bmod 4) .\end{cases}$

It can be verified in similar lines as in Case 1 that $f$ is a total unidominating function.

$$
\begin{aligned}
& \operatorname{Now} \sum_{u \in V} f(u)=\underbrace{0+1+1+0}+\underbrace{0+1+1+0}+\cdots \\
&+\underbrace{0+1+1+0}+0+1+1 \\
&=2\left(\frac{n-3}{4}\right)+2=\frac{n+1}{2}=\left\lceil\frac{n}{2}\right\rceil .
\end{aligned}
$$

Therefore $\gamma_{t u}\left(P_{n}\right) \leq\left\lceil\frac{n}{2}\right\rceil---(1)$

Now $n \equiv 3(\bmod 4) \Rightarrow n-3 \equiv 0(\bmod 4)$.
Then as per the discussion in Case 1, it can be seen that any total unidominating function $f$ has functional values sum for the vertices $v_{1}$ to $v_{n-3}$ as

$\sum_{i=1}^{n-3} f\left(v_{i}\right) \geq \frac{n-3}{2}$

As per the discussion in Case 3, if $f$ is a total unidominating function of $P_{n}$ with minimum weight then

$\sum_{i=n-2}^{n} f\left(v_{i}\right)=2$.

Therefore $f(V)=\sum_{u \in V} f(u)=\sum_{i=1}^{n-3} f\left(v_{i}\right)+\sum_{i=n-2}^{n} f\left(v_{i}\right)$

$$
\geq \frac{n-3}{2}+2 \geq \frac{n+1}{2} \geq\left\lceil\frac{n}{2}\right\rceil \text {. }
$$

Thus $\gamma_{t u}\left(P_{n}\right) \geq\left\lceil\frac{n}{2}\right\rceil---(2)$

Therefore from the inequalities (1) and (2) $\gamma_{t u}\left(P_{n}\right)=\left\lceil\frac{n}{2}\right\rceil$. Thus for all possibilities of $n$,it is proved that

$$
\begin{gathered}
\gamma_{t u}\left(P_{n}\right)=\left\lceil\frac{n}{2}\right\rceil \text { if } n \equiv 0,1,3(\bmod 4) \\
\text { and } \gamma_{t u}\left(P_{n}\right)=\left\lceil\frac{n}{2}\right\rceil+1 \text { if } n \equiv 2(\bmod 4) \text {. }
\end{gathered}
$$

Theorem 3.2: The number of total unidominating functions of $P_{n}$ with minimum weight is

$$
\begin{cases}1 & \text { whenn } \equiv 0(\bmod 4), \\ \left\lfloor\frac{n}{4}\right\rfloor & \text { when } n \equiv 1(\bmod 4), \\ \left\lfloor\frac{n}{4}\right\rfloor+\frac{1}{2}\left\lfloor\frac{n}{4}\right\rfloor\left(\left\lfloor\frac{n}{4}\right\rfloor-1\right)+1 & \text { whenn } \equiv 2(\bmod 4), \\ 2 & \text { whenn } \equiv 3(\bmod 4) .\end{cases}
$$

Proof: Let $P_{n}$ be a path with vertex set $V=\left\{v_{1}, v_{2}, \ldots, v_{n}\right\}$.

Now the number of total unidominating functions with minimum weight $\gamma_{t u}\left(P_{n}\right)$ is found in the following four cases.

Case 1: Let $n \equiv 0(\bmod 4)$.

The function $f$ defined in Case 1 of Theorem 3.1 is given by

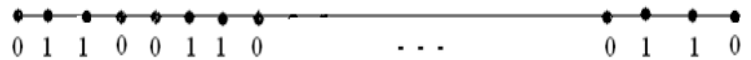

The functional values of $f$ are $01100110---0110$.

Take $a-0110$. Then the functional values of $f$ are in the pattern of $a a a \ldots a$. (here there are $\frac{n}{4} a^{\prime} s$ ).

By arranging these letters in different ways, we get different total unidominating functions with the same weight which is minimum as per Theorem 3.1. But these letters $a a a \ldots a$ can be arranged in one and only one way so that there is one and only one total unidominating function with minimum weight.

Case 2: Let $n \equiv 1(\bmod 4)$.

The function $f$ defined in Case 2 of Theorem 3.1 is given by

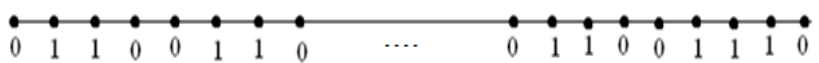

The functional values of $f$ are $01100110---011001110$ 
Take $a-0110, b-01110$. Then the functional values of $f$ are in the pattern of $a a a \ldots a b$ (here there are $\frac{n-5}{4} a^{\prime} s$ ). By arranging these letters in different ways, different total unidominating functions with minimum weight $\left\lceil\frac{n}{2}\right\rceil$ are obtained. As there are $\frac{n-5}{4} a^{\prime} s$ and one $b$, these letters $a^{\prime} s$ and $b$ can be arranged in $\frac{\left(\frac{n-5}{4}+1\right) !}{\left(\frac{n-5}{4}\right) ! \cdot 1 !}=\frac{\left(\frac{n-1}{4}\right) !}{\left(\frac{n-5}{4}\right) !}=\frac{n-1}{4}$ ways. Therefore there are $\frac{n-1}{4}=\left\lfloor\frac{n}{4}\right\rfloor$ total unidominating functions with minimum weight.

Case 3: Let $n \equiv 2(\bmod 4)$.

The function $f$ defined in Case 3 of Theorem 3.1 is given by

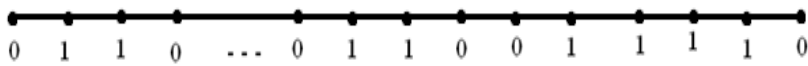

The functional values of $f$ are $01100110 \ldots 0110011110$.

Take $a-0110, c-011110$. Then the functional values of $f$ are in the pattern of $a a a$... $a c$ (here there are $\frac{n-6}{4} a^{\prime} s$ ). As in Case 2 these letters can be arranged in $\frac{n-2}{4}$ ways.

Therefore there are $\frac{n-2}{4}$ total unidominating functions with minimum weight.

Now some other total unidominating functions with the same weight and which are not covered in the above set of functions will be investigated.

Define a function $f_{1}: V \rightarrow\{0,1\}$ by

$$
f_{1}\left(v_{i}\right)=f\left(v_{i}\right) \forall v_{i} \in V \text {, except for } i=n-4, i=n-6
$$

and $f_{1}\left(v_{n-4}\right)=0, f_{1}\left(v_{n-6}\right)=1, n \geq 10$.

It can be easily verify that the function $f_{1}$ is also a total unidominating function.

Further,

$$
\begin{aligned}
\sum_{u \in V} f_{1}(u)= & \underbrace{0+1+1+1+0}_{+\underbrace{0+1+1+1+0}+\cdots+\underbrace{0+1+1+0}+1+0} \\
& =\frac{2(n-10)}{4}+3+3=\frac{2 n+4}{4}=\frac{n}{2}+1 .
\end{aligned}
$$

The function $f_{1}$ is given by

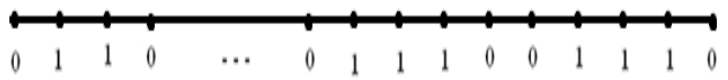

The functional values of $f_{1}$ are $01100110 \ldots 01100111001110$.

Take $a-0110, b-01110$. Then the functional values of $f_{1}$ are in the pattern of $a a a \ldots a b b$. Here there are $\frac{n-10}{4} a^{\prime} s$ and two $b^{\prime} s$. These $\frac{n-10}{4}+2=\frac{n-2}{4}$ letters can be arranged in

$\frac{\left(\frac{n-2}{4}\right) !}{\left(\frac{n-10}{4}\right) ! \cdot 2 !}=\frac{1}{2}\left(\frac{n-2}{4}\right)\left(\frac{n-6}{4}\right)$ ways. Therefore there are

$\frac{1}{2}\left(\frac{n-2}{4}\right)\left(\frac{n-6}{4}\right)$ total unidominating functions with minimum weight $\left\lceil\frac{n}{2}\right\rceil$.

Another function $f_{2}: V \rightarrow\{0,1\}$ is defined by

$$
f_{2}\left(v_{i}\right)=\left\{\begin{array}{cc}
1 & \text { for } i \equiv 1,2(\bmod 4), \\
0 & \text { otherwise }
\end{array}\right.
$$

As in the previous cases, it canbe easily prove that $f_{2}$ is a total unidominating function.

$$
\text { Further } \begin{aligned}
\sum_{u \in V} f_{2}(u) & =\underbrace{1+1+0}+\underbrace{0+1+1+0}+\cdots \\
& +\underbrace{0+1+1+0}+\underbrace{0+1+1} \\
& =2+\frac{2(n-6)}{4}+2=\frac{2 n-12}{4}+4 \\
& =\frac{2 n+4}{4}=\frac{n}{2}+1 .
\end{aligned}
$$

The function $f_{2}$ is given by

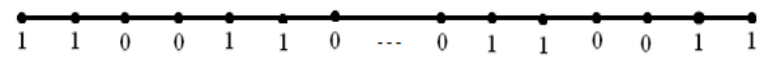

The functional values of $f_{2}$ are $1100110---0110011$ Take $a-0110$. Then the functional values of $f_{2}$ are in the pattern of $110 a a a \ldots a 011$.

These letters $a, a, \ldots, a$ can be arranged in only one way. Therefore there is only one total unidominating function. Thus there are $\frac{n-2}{4}+\frac{1}{2}\left(\frac{n-2}{4}\right)\left(\frac{n-6}{4}\right)+1=\left\lfloor\frac{n}{4}\right\rfloor+$ $\frac{1}{2}\left\lfloor\frac{n}{4}\right\rfloor\left(\left\lfloor\frac{n}{4}\right\rfloor-1\right)+1$ total unidominating functions with minimum weight $\left\lceil\frac{n}{2}\right\rceil+1$.

Case 4: Let $n \equiv 3(\bmod 4)$.

The function $f$ defined in Case 4 of Theorem 3.1 is given by

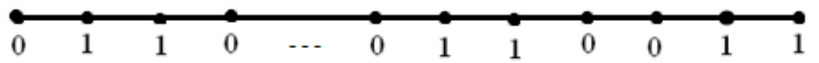

The functional values of $f$ are $01100110---0110011$. Take $a-0110$. Then the functional values of $f$ are in the pattern of $a a a \ldots a 011$ (here there are $\frac{n-3}{4} a^{\prime} s$ ). As the letters $a a \ldots a$ can be arranged in only one way, there is one and only one total unidominating function with minimum weight $\left\lceil\frac{n}{2}\right\rceil$.

Now another function $f_{1}: V \rightarrow\{0,1\}$ is defined by

$f_{1}\left(v_{i}\right)=\left\{\begin{array}{lr}1 & \text { for } i \equiv 1,2(\bmod 4) \\ 0 & \text { otherwise }\end{array}\right.$

Then on similar lines of Case 3 we can show that $f_{1}$ is a total unidominating function.

$$
\begin{aligned}
& \text { Further } \sum_{u \in V} f_{1}(u)=\underbrace{1+1+0}+\underbrace{0+1+1+0} \\
& +\cdots+\underbrace{0+1+1+0} \\
& =2+\frac{2(n-3)}{4}=\frac{2 n+2}{4}=\frac{n+1}{2}=\left\lceil\frac{n}{2}\right\rceil .
\end{aligned}
$$

The function $f_{1}$ is given by

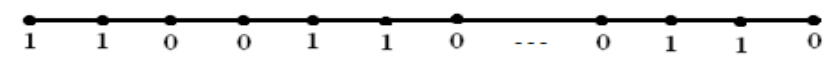

The functional values of $f_{1}$ are1100110 - - 0110 .

Take $a-0110$. Then the functional values of $f_{1}$ are in the pattern of 110 aaa ... a. 
The letters $a a \ldots a$ can be arranged in only one way. Therefore there is only one total unidominating function.

Thus there are two total unidominating functions for $P_{n}$ with minimum weight $\left\lceil\frac{n}{2}\right]$

\section{ILLUSTRATIONS}

Example 4.1: Let $\boldsymbol{n}=24$.

Obviously $24 \equiv 0(\bmod 4)$.

The functional values of a total unidominating function $f$ defined in Case 1 of Theorem 3.1 are given at the corresponding vertices of $P_{24}$.

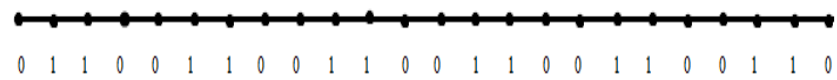

Total unidomination number of $P_{24}$ is $\gamma_{t u}\left(P_{24}\right)=\left\lceil\frac{24}{2}\right\rceil=12$.

There is only one total unidominating function $f$ with minimum weight 12 .

\section{Example 4.2: Let $n=33$.}

Clearly $33 \equiv 1(\bmod 4)$.

The functional values of a total unidominating function $f$ defined in Case 2 of Theorem 3.1 are given at the corresponding vertices of $P_{33}$.

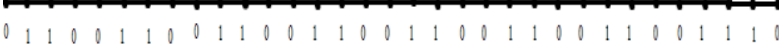

The total unidomination number of $P_{33}$ is $\gamma_{t u}\left(P_{33}\right)=\left\lceil\frac{33}{2}\right\rceil=17$. There are $\left\lfloor\frac{n}{4}\right\rfloor=\left\lfloor\frac{33}{4}\right\rfloor=8$ total unidominating functions with minimum weight 17 .

\section{Example 4.3: Let $n=42$.}

Obviously $42 \equiv 2(\bmod 4)$.

The functional values of a total unidominating function $f$ defined in Case 3 of Theorem 3.1 are given at the corresponding vertices of $P_{42}$.

011001100110011001100111001100111001100111110

Total unidomination number of $P_{42}$ is

$\gamma_{t u}\left(P_{42}\right)=\left\lceil\frac{n}{2}\right\rceil+1=\left\lceil\frac{42}{2}\right\rceil+1=22$.

There are $\left\lfloor\frac{n}{4}\right\rfloor=\left\lfloor\frac{42}{4}\right\rfloor=10$ total unidominating functions with minimum weight 22 .

The functional values of another total unidominating function $f_{1}$ defined in Case 3 of Theorem 3.2 are given at the corresponding vertices of $P_{42}$.

011001100110011001100110001100110011100
There are $\frac{1}{2}\left\lfloor\frac{42}{4}\right\rfloor\left(\left\lfloor\frac{42}{4}\right\rfloor-1\right)=\frac{10 \times 9}{2}=45$ total unidominating functions with minimum weight 22 .

The functional values of another total unidominating function $f_{2}$ defined in Case 3 of Theorem 3.2 are given at the corresponding vertices of $P_{42}$.

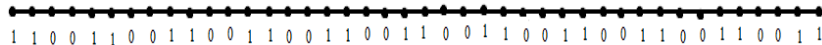

Only one total unidominating function exists with minimum weight 22 .

Thus there are $10+45+1=56$ total unidominating functions of $P_{42}$ with minimum weight 22 .

Example 4.4: Let $n=19$

Clearly $19 \equiv 3(\bmod 4)$.

The functional values of a total unidominating function $f$ defined in Case 4 of Theorem 3.1 are given at the corresponding vertices of $P_{19}$.

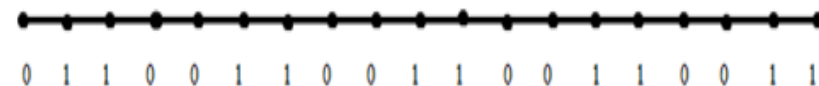

The total unidomination number of $P_{19}$ is

$\gamma_{t u}\left(P_{19}\right)=\left\lceil\frac{19}{2}\right\rceil=10$.

There is only one total unidominating function with minimum weight 10 .

The functional values of another total unidominating function $f_{1}$ defined in Case 4 of Theorem 3.2 are given at the corresponding vertices of $P_{19}$.

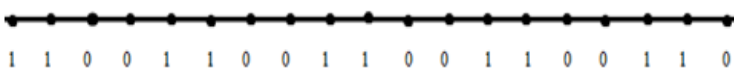

There is only one total unidominating function with minimum weight 10 .

Therefore there are $1+1=2$ total unidominating functions with minimum weight 10 .

\section{CONCLUSION}

It is interesting to study the new concepts introduced unidominating functions and total unidominating functions for various graphs. The authors made an attempt to study these concepts for some standard graphs and obtained the results. This work enhances the study of unidominating functions, total unidominating functions and total unidomination number of a cycle. The authors have also studied these concepts for cycles and obtained the results. 


\section{REFERENCES}

[1] Allan, R.B. and Laskar, R.C. (1978), On domination, independent domination numbers of a graph, Discrete Math., 23, 73-76.

[2] Anantha Lakshmi, V and Maheswari, B. -Unidominating functions of a Path, IJCET, volume 6(2015), 11-19.

[3] Cockayne, E.J. and Hedetniemi, S.T. (1977), Towards a theory of domination in graphs, Networks, 7, 247-261.

[4] Cockayne, C.J., Dawes, R.M., and Hedetniemi, S.T., Total domination in graphs, Networks, 10 (1980), 211 219.
[5] Cockayne, E.J., Mynhardt, C.M. and Yu, B. Total dominating functions in trees: Minimality and Convexity, Journal of Graph Theory, 19(1995), 83-92.

[6] Haynes, T.W. Hedetniemi, S.T. Slater, P.J. Fundamentals of domination in graphs, Marcel Dekker, Inc., New York (1998).

[7] Haynes, T. W., Hedetniemi, S.T., $\quad$ Slater, P.J., Domination in graphs: Advanced Topics, Marcel Dekker, Inc., New York (1998). 